Hans-Christian STILLMARK

https://orcid.org/0000-0002-9569-998X

Universität Potsdam

\title{
Über das verbotene Lachen
}

\section{About the forbidden laughter}

\begin{abstract}
The essay explores the question of what happens in forbidden contexts when laughter cannot be suppressed. A childhood experience is the occasion to reflect on the communicative relations of forbidden laughter. Laughter is pointed out as a special communicative sign system and physical act.
\end{abstract}

Keywords: theatre, phylogenesis, taboo, punishment, observations (Luhmann), carnival, malice.

Dass das Lachen mit mehrfachen Grenzüberschreitungen zu tun hat, ist bekannt. Dass das Lachen daher in autoritären Strukturen Gefahr läuft zu den Subversionen zu gehören, die verfolgt, verboten und bestraft werden, ist auch unstrittig. Die karnevalistische Lachkultur, Hohn und Häme eines Thersites und andere vom Lachen heraufbeschworene Konflikte gehören zum Inventar menschlicher Kultur wahrscheinlich seit es hierarchische Schichtungen innerhalb der menschlichen Sozietät gibt. An die Anfänge des menschlichen Lachens in der anthropologischen Kultur kann ich nicht zurückgehen. Im Tierreich scheint es Anläufe zum Lachen zu geben. Sie mögen an die Abwesenheit von Not gebunden sein und markieren in gewisser Weise Lust am Spiel, auch am Zusammenspiel mit Artgenossen. Vieles ist hier noch unerforscht, ungeklärt und hypothetisch. Um diesen Unsicherheiten zu entgehen, will ich ein Erlebnis schildern, das vielleicht nicht unbedingt zur menschlichen Phylogenese, so doch zu meinem ontogenetischen Werdegang gehört. 
An der Schwelle des letzten Drittels meines Lebens und eingedenk der kulturwissenschaftlichen Wende, ist es wohl erlaubt derart ins Anekdotische zurückzugreifen, um mich verständlich zu machen.

Aufgewachsen bin ich in einer Welt, in der mehrere einander bekämpfende Wertsetzungen ihre Gültigkeit beanspruchten, um es auf meinen Fall zu beziehen: Das Ereignis trug sich im sich „auf dem Sprung zum Kommunismus" befindlichen Teil Deutschlands zu, etwa in der Mitte der 60er Jahre eine Zeit, die nach dem Mauerbau als Kalter Krieg bezeichnet wurde. Wie alle in der „Blumenstadt" Erfurt Geborenen bin ich eine „Puffbohne“. In meiner Geburtsstadt, die damals eine Bezirkshauptstadt war, sind im Verlaufe der Geschichte unterschiedlichste Werte zu Stein geworden. Dom und Severikirche beherrschen den wichtigsten Markt der Innenstadt, Katholizismus und Protestantismus schauen auf die Bevölkerung herab. Der daneben aufragende Petersberg mit seiner Burg dagegen weiß von Rüstungen weltlicher Mächte. Meine 1961 eingeweihte Schule trug den Namen des ersten Menschen, der ins Weltall geschossen wurde: Juri Gagarin. Den Sämereien und Blumensorten, die man in der Blumenstadt Erfurt züchtete, sind ihre ideologischen Herrschaftsbegehren nicht anzusehen. Die ideologischen Begehren haben sich vielmehr in den Namen verborgen. Man kennt das Vergissmeinnicht, ebenso wie die Kaiserkrone, die Narzisse, wie die Schwertlilie, Rittersporn wetteifert mit der symbolgewordenen Rose. Ob es eine Züchtung namens „Sputnik“ gibt, ist nicht auszuschließen - in den Samenverlagen meiner Heimatstadt ist gewiss noch viel, viel mehr zu entdecken, was von der Überwölbung der Natur durch die menschliche Sprache und Zivilisation zeugt.

Mein Erlebnis mit dem verbotenen Lachen, das mir bis heute plastisch vor Augen steht, trug sich im Alter von etwa 12 oder 13 Jahren zu. Um die Rollenspiele, die unserem (theatralischen) Leben zugehörig sind, nur anzudeuten: ich war damals ein Pionier in der Schule, ein gehorsamer Sohn meiner Eltern, die selbst wiederum sich sehr unterschiedlichen Wertordnungen verpflichtet fühlten. Während mein Vater Schauspieler, Leiter des Kindertheaters und Abgeordneter des Kulturbundes in der Stadtverordnetenversammlung war, ging meine Mutter in die von Rudolf Steiner inspirierte "Christengemeinschaft". Es gehört zu den Ungereimtheiten der „Diktatur des Proletariats" auf deutschem Boden, dass die Religionsausübung nach der Steiner'schen Lehre erlaubt, die Gesellschaft der Anthroposophie jedoch verboten war. Mir machte dieser Konflikt seinerzeit nicht viel aus. Ich ging jeden Sonntag zum Kindergottesdienst, war in der Schule „Für Frieden und Sozialismus - Immer bereit!", übte mit mehr oder weniger Elan auf dem Cello und probierte mich in den unterschiedlichsten Sportarten aus. Als jüngstes Kind meiner Eltern hatte ich hin und wieder kleine Rollen auf der Bühne zu 
spielen, die Verstellung und Angleichung in der Welt der Großen und Kleinen fiel mir nicht schwer.

Die „Christengemeinschaft" hatte lange in Erfurt kein richtiges Kirchengebäude. Sie befand sich seit Kriegsende in einem mehrstöckigen bürgerlichen Wohnhaus in der Herderstraße. So kam es, dass wir in der Familie nicht etwa sagten: „Ich gehe in die Kirche“, es hieß vielmehr (vielleicht auch um Reibungspunkte zu vermeiden): „Ich geh in die Herderstraße“. Die Bezeichnung Villa für das dortige Haus wäre zu hochtrabend für den Zweietagenbau, aber erwähnenswert ist doch, dass im Erdgeschoss dieses Hauses ein Altarraum, eine Sakristei und ein Gemeinderaum sowie eine kleine Bühne mit Orgel existierte. In der oberen Etage waren die Wohnungen (eher Zimmer) der meist alleinstehenden Pfarrer, sehr beengt auf kleinstem Raum zu finden.

Der Kindergottesdienst wurde sonntags nach der „Menschenweihehandlung" zelebriert. Er war für Kinder im Alter von ca. 5 bis 14 Jahren konzipiert und lief nach einem festen Ritual mit großem Gleichmaß unverändert ab. Den Kindern wurde auf diese Weise die Heilige Schrift nahegebracht. Sie erlebten im Wechsel der Altarfarben das kirchliche Jahr. Für sie wurden die Kerzen des Altars entzündet und zum Schluss gelöscht. Dazwischen lag in einem Zeitraum von ca. zehn Minuten die „Kinderhandlung“1.

In meiner Erinnerung hatte sich vor allem eine Pfarrerin auf die „Kinderhandlung" spezialisiert. Sie wurde aber auch bei Bedarf durch andere Pfarrer vertreten. Und so kam es, dass an dem Tag, wo das verbotene Lachen mir eindringlich bewusst wurde, der Kindergottesdienst durch den greisen Herrn S. durchgeführt wurde. Wir Kinder kannten die Pfarrer lediglich oberflächlich und wir machten uns über sie höchst selten wirklich Gedanken. Unzweifelhaft hatten die Pfarrer über die Gemeinde eine uneingeschränkte und große Autorität. Ihnen gehörte auch fraglos unser kindlicher Respekt in vollem Maße. Über Herrn S. wussten wir Kinder wenig. Uns war klar, dass auch er ein Zimmer im Hause bewohnte und dass er alt und auch schon hinfällig war. Aus heutiger Sicht würde ich behaupten, Herr S. hatte zumindest einen Schlaganfall leidlich überstanden. Sein Geh- und sein Sprachvermögen waren beeinträchtigt. Eine gewisse Maskenhaftigkeit ließ ihn immer gleich aussehen. Er hörte schwer und sprach recht undeutlich. Wir waren am besagten Tag vier Kinder, die zum Gottesdienst erschienen waren. Außer mir waren der gleichaltrige Michael und zwei weitere jüngere Kinder an jenem Sonntag in der Kinderhandlung. Die Jüngeren waren ein Brüderpaar, das wir „Großen“ respektlos unter uns „Wackelkopf" und „Bratkartoffel“ nannten. Die Spitznamen mögen anzeigen, dass uns Kindern der Spaß nicht mit dem Ernst

1 Ausführlicher über das Ritual der sonntäglichen Kinderhandlung siehe: https://anthrowiki.at/Der_freie_christliche_Impuls\#Sonntagshandlung_f.C3.BCr_die_Kinder.5B31.5D 
des Gottesdienstes auszutreiben war. Ob wir am besagten Tage schon mit Scherzen und anderen Lachhaftigkeiten gut vorgesorgt hatten, kann ich nicht mehr erinnern. Ich nehme aber an, wir hatten. Mit Michael war gut Spaß zu haben und eine Freude war für mich das sonntägliche Wiedersehen mit ihm. Die Gleichförmigkeit des Rituals und die relative Tristesse des pflichtgemäßen Kirchenganges wurde dadurch ein wenig aufgehoben. Vermutlich einigermaßen angegackert überstanden wir den ersten Teil der Zeremonie. Dazu hatten wir dem Ministranten die Hand zu geben und uns die immer gleichen Worte anzuhören, dass wir jetzt zur Kinderhandlung eingelassen werden, dort das Wort Gottes zu hören sein werde, dass wir unsere Herzen öffnen sollen usw. Wir hatten uns dann am Rande des Altarteppichs aufzustellen und der Dinge zu harren, die da auf uns zukamen. Dieses Mal also in Gestalt von Herrn S. Der schlurfte langsam heran und es war schon ein Glück, dass er nicht an den wenigen Stufen zum Altar das Gleichgewicht verlor. Herr S. kam uns unglaublich alt vor, seine Artikulation war kaum zu verstehen. Bei seinen ersten Lauten, sah ich aus den Augenwinkeln Michael neben mir und im gleichen Augenblick durchfuhr uns beide ein spontaner Lachreiz. Krampfartig zog es uns zusammen, wir mussten uns noch bestimmter voneinander abwenden, aber es war nun einmal geschehen, der Lachreiz steigerte sich. Worüber wir lachten, lag auf der Hand und war gleichzeitig völlig unbegriffen. Sehr wahrscheinlich lachten wir auch, über unser Lachen. Aber ich will derartigen Unbestimmtheiten nicht vorgreifen. Es sei lediglich betont, dass es sich nicht um ein einfaches Auslachen handelte, es lagen da noch ganz andere Zusammenhänge vor, die uns jedoch nicht klar waren. Je mehr wir aber das Lachen bekämpften und niederzuhalten versuchten, umso stärker war der Zwang dem Lachen nachzugeben. Es war wie verhext und kaum auszuhalten. Vor uns die erhobenen Arme des Pfarrers, der mit den Worten kämpfte, hinter uns im Gemeinderaum wussten wir ein paar Zuschauer, die uns sicher zusahen... es war unerträglich und wir hätten uns vor Lachen wälzen mögen. Während Herr S. mit geschlossenen Augen seinen unverständlichen Text lallte, beruhigten wir uns einen Augenblick, sahen aber zueinander und die nächste Lachkanonade schüttelte uns noch kräftiger durch. Nach einer gefühlten Ewigkeit, in der wir mit Tränen in den Augen und schniefenden Nasen voneinander wegsahen, uns das Lachen, so gut es ging, verbissen und doch immer wieder von Zuckungen überwältigt wurden, ging die Kinderhandlung zu Ende. Kaum draußen, mussten wir an die frische Luft, um uns endlich befreit auszuschütten, da bekam ich noch in der Tür einen Schlag von hinten an den Kopf. Ein wütender älterer, bereits konfirmierter Junge hatte mich als Anstifter und Missetäter identifiziert und strafte mich also ab. Dass er das Amt der Gerechtigkeit auf diese Weise vertrat, konnte ich ihm weder verzeihen noch vergessen. 
Dass dieser Büttel Gottes später die Priesterlaufbahn in der „Christengemeinschaft" einschlug, stellte für mich alles in Frage. Nach diesem Schrecknis war meine Gottesfurcht aufgebraucht. Die Ohrfeige à la Makarenko² hatte mich nicht zu einem besseren Menschen bekehrt. Im Gegenteil, ich fiel für den Rest meines Lebens recht deutlich vom Glauben. Aber da beginnen andere Geschichten, die ich hier nicht erzählen möchte.

Bemerkenswert an dem Vorfall ist der Aufbau, der sich wie eine Versuchsanordnung lesen lässt. Da ist zum ersten die theatralische Situation, die die Szene konstituiert. Wir Kinder sind Zuschauer des Pfarrers, dieser hat uns ungeachtet der geschlossenen Augen natürlich im pädagogischen Blick und hinter uns in unserem Rücken wissen wir eine kleine, in Andacht versunkene ernste Gemeinde, die dem Geschehen zuschauend beiwohnt. Wir fühlen uns also unter doppelter Beobachtung, wenn man so will eigentlich unter dreifacher Beobachtung, da wir uns ja selbst auch sehen und wahrnehmen. Diese Vielzahl beobachtender Instanzen, die für Niklas Luhmann und seine systemtheoretische Schülerschaft bestimmt eine seminaristische Studienaufgabe wert sein könnten, war uns in Moment der Ereignisse nicht klar. Fest stand, wir fühlten uns beobachtet, sonst hätten wir uns ja frei bewegt und im Lachen nicht stören lassen. Das Verhexte an der Situation war jedoch, dass wir einem Zwang zum Lachen ausgesetzt waren, der sich nicht überspielen ließ. Auch das gegenseitige Anspornen durch die lachhafte Partnerschaft war unabweisbar. „Lachen steckt an“ - diese sprichwörtliche Weisheit erlebten und erlitten wir damals in vollem Maße.

Vielleicht muss hier noch einmal daran erinnert werden, dass wir Menschen das Lachen bereits erlernen und äußern bevor wir eine Sprache sprechen. Vermutlich verstehen wir im Säuglingsalter bereits etwas von dem, was unsere Eltern insbesondere die Mutter äußert, aber über das Lachen bildet visuell ein sehr frühes sozialkommunikatives Band mit anderen Menschen. Nach Luhmann ist ja Kommunikation mit Gesellschaft identisch. Die Mimik ist so eine sehr frühe Grundierung unseres sozialen Seins. Es bindet uns in die Familie ein. Es ist nebenbei auch bedenkenswert, dass das Lachen bei allen Völkern etwa gleich ist, unabhängig von den später sehr unterschiedlich erlernten Sprachen und Lautungen. Beginnt es nicht zunächst als stummes Zeichen, noch ohne akustische Untermalung? Ich beginne hier zu stocken, da ich diesbezügliche Studien nicht kenne, aber ich folge doch der Hypothese, dass die Kleinsten, noch bevor sie sprechen, stumm lächeln können. Es gibt in der deutschen Sprache für dieses frühe mimische Zeichen ei-

2 Anton Semjonowitsch Makarenko war der vorbildliche sowjetische Pädagoge nach dessen Beispiel die Erziehung zum Kommunismus gemeistert werden sollte. Sein Buch Der Weg ins Leben gehörte zur Pflichtlektüre für die sozialistisch zu Erziehenden. 
nen lexikalischen Ausdruck - das Engelslächeln. Dieses ist noch ungerichtet und erscheint fast zufällig im Antlitz des Säuglings. Die Mutter, wie auch die anderen Betrachter reagieren jedoch darauf. Sie sprechen das Kind mit dem Ausdruck des Entzückens an, heben dabei die Stimmen, signalisieren so einen dem Säugling zugewandten Zustand, der irgendwann verstanden und rückgekoppelt wird. Es bildet sich auf diese Weise sukzessive eine kommunikative Struktur heraus, die sich durch Wiederholung festigt. Das in der weiteren Entwicklung beobachtbare zielgerichtete Lächeln, das auch bald durch heftige Gesten sowie durch die Stimme unterstützt wird, zeigt ein kleines Kind, das mit dem ganzen Körper aktiv sich beim Lachen äußert. Das Strampeln, das stoßweise Atmen ist im Modus des Vergnügens zu erkennen, die Voraussetzungen des Lachens bilden sich heraus. Wir alle, die mit Kindern aufwachsen, kennen diese Szenen und das davon begleitete Entzücken, welches unsere soziale Bindung zu dem lachenden Kind immer stärker festigt.

Halten wir fest: Das Lachen entwickelt sich vom zufälligen (oder vielleicht doch genetisch angelegten?) Grimassieren zu einer zielgerichteten kommunikativen Handlung, die den ganzen Körper erfasst. Lachen will gelernt sein. Es steht den frühesten menschlichen Äußerungen so nahe wie das Weinen. Was begründet aber diese Äußerung, die nur dann Sinn hat, wenn sie an andere Menschen gerichtet wird? Auch hier kann ich nur vermuten, sie ist an die Versorgung mit Nahrung gebunden, auch dies als unterstützendes soziales Band, um auch beim nächsten Hungergefühl nicht vergessen zu werden. Dass sich zu der Funktion, die Versorgung mit Nahrung zu unterstützen, im Verlauf der Kindheit noch weitere Sinnbezüge an das Lachen amalgamieren, scheint mir ebenso evident. Die kulturellen Aussagen des Lachens nehmen dabei im Laufe der jeweiligen individuellen und kollektiven Entwicklung immer neue Semantiken an. In diesem Überschuss sind die frühen ungerichteten Handlungen wie die aktuellen zielgerichteten aufgehoben. Sie sind nur theoretisch zu trennen, in unsere Praxis des Lachens wirken sich komplex, unüberschaubar und nur schwer kalkulierbar.

Auch ohne die Sprache zu verstehen, werden wir vom Lachen der anderen ergriffen, vom Weinen ohnehin. Möglicherweise ist dieses frühe Vermögen, das das Lachen hervorbringt und immer wieder erneut eingeübt hat, dafür verantwortlich, dass wir mit anderen lachen, bevor wir den Witz oder den Grund des Lachens überhaupt kennen. Wenn wir den jeweiligen Grund erfahren, kann es sogar sein, dass „uns das Lachen vergeht“...

Lässt man sich die theatralische Verfassung dieses Ereignisses noch länger durch den Kopf gehen, so ist der Zuschauer, der wir auch in unserem Beispiel waren, zum Hauptakteur des Lachens verwandelt worden. Ein Rol- 
lenwechsel., analog zu dem, der bei Bachorski beschrieben wurde, ${ }^{3}$ hatte also stattgefunden und die bisherigen Handlungsvorschriften hatten sich unversehens gewandelt. Um es anzudeuten: vom disziplinierten Kind, das den gottesdienstlichen Vorschriften stumm Folge leistet, wurden wir zu Akteuren unseres Lachanfalls, der die bisherigen Normen beiseite fegte. War das Lachen in diesem Rahmen schon Ketzerei? Kein Verbot und kein Gebot hatten die Kraft uns in unserem Tun abzuhalten oder zu unterbrechen. Eine stumme Macht hatte sich an die Stelle der priesterlichen Autorität gesetzt, es war die Macht des im Lachen sich manifestierenden Komischen. Anders aber als in Andersens Märchen von des „Kaisers neuen Kleidern“ steckten wir mit unserem Lachen nicht „das ganze Volk“ zum Mitlachen an, sondern wir säten Empörung über unsere Respektlosigkeit.

Dennoch bleibt festzuhalten, dass ein theatralischer Rollenwechsel mit dem Lachen einhergeht. Vom zuschauenden Beobachter zum aktiven Verarbeiter einer Wertsetzung wandeln sich beim Lachen die Rollen. Gleichwohl: Bemerkt man die Unangemessenheit des Lachens in der aktuellen Situation, so vermeidet man es und weicht aus. Man spielt dann eine Oblomov'sche Gleichmütigkeit, die nichts aus der Ruhe bringen kann.

In meinem Erlebnis konnte die rettende Gleichmut nicht erreicht werden. Das Verbot, das das Lachen in die Schranken weisen sollte, stellte sich als keine wirkliche Grenze heraus. Das Lachen, es entgrenzte sich in einem Kontext, wo es als Grenzverletzung, und hier eigentlich schon als Tabubruch sich freisetzte. Eine nicht unbedeutende Rolle spielt die Plötzlichkeit des Reizes, der das Lachen in Gang setzt. Das Überraschende, Spontane, Unwillkürliche und Zugespitzt-Pointierte das zur Reaktion des Lachens führt, überführt den ganzen Organismus von der Ruhe in die energische Bewegtheit. Und das wäre eine nächste Bestimmung: Der Körper des zum Lachen Gezwungene ist dynamisch bewegt. Er befreit sich von den selbst oder fremd auferlegten Grenzen und drängt zur Äußerung. So wird der Lachende in einen dynamischen Prozess der Selbstveränderung gebracht und zeigt diese Veränderung als Verhaltensänderung. Das Verhalten ist in der körperlichen Dynamik des Lachvorgangs ein komplexes Geschehen. Es lassen sich die daran beteiligten Organe und Gruppen hier nennen, sie zu beschreiben übersteigt meine anatomischen Kenntnisse sowie die sprachlichen Möglichkeiten. Es sind Studien zufolge über 300 Muskeln in dynamischen Prozessen bewegt, die Atmung vertieft sich, Verspannungen lockern sich, der Blutdruck sinkt, Endorphine, sogenannte Glückshormone werden ausgestoßen. Bei dem unterdrückten Lachen werden Blutdruck und Endorphine wohl nicht

3 Vgl. Hans-Jürgen Bachorski, „Poggios Facetien und das Problem der Performativität des toten Witzes“, Paragrana, Nr. 10 (2001): 318-335. 
freigesetzt werden können. Sie sind, wie auch die Atmung gestaut und verursachen, wie ich mich erinnere krampfartige Zustände. Beim ungehinderten freigesetzten Lachen hingegen soll im Fall eines einigermaßen sanguinischen Charakters die Lebenszeit um sieben (!) Jahre verlängert werden können. All das vollzieht sich im Lachvorgang, ohne dass wir mit unserem Willen dies vollführen könnten, es geschieht als hätte uns eine fremde Macht ergriffen. Dass der ganze Bewegungsapparat vom Lachen ergriffen ist, zeigt die Gestik und Mimik an. Und selbst gegen unseren Willen, also im unterdrückten Modus kann sich das Lachen mit seinen unerlaubten Zeichen verraten. Sie sind suspekt und nicht zu hintergehen. Die Stimme und ihre Kontexte, die mimischen und gestischen Bewegungen, die sich deutlich vom Zustand der Gleichmütigkeit abheben, zeigen den Lachenden durch seinen verräterischen Körper.

Als ein nach außen lesbarer Prozess - selbst das unterdrückte Lachen aus unserem Beispiel hat für die äußeren Beobachter und Beteiligten eine Signalwirkung - bedeutet das Lachen eine gegenüber der Umwelt wertende Stellungnahme. Damit hat sich eine weitere Determination aufgemacht, die Wertung lässt sich im geschilderten Fall von außen betrachtet als Abwertung lesen. Der Lachende ordnet sich in unserem Beispiel (scheinbar) in seiner sozialen Hierarchie über das Objekt seines Vergnügens. Er nimmt sich das Recht, so sah es auf jeden Fall der zur späteren Strafe Ermächtigte, ein vernichtendes Urteil offen zu zeigen.

Dass das Lachen bei den herangewachsenen Kindern damals ein derartig klares abwertendes Urteil darstellte, würde ich bezweifeln. Es mischten sich noch einige andere Veranlassungen und Gründe in die Situation, die sich eher als verworren, denn als klar les- und verstehbar erwies.

Um aus der Gemengelage einiges anzudeuten: Dass der Lachreiz uns gleichzeitig ergriff, dass wir plötzlich gemeinsam lachten, dass das Lachen den manchmal unerträglichen Ernst der Situation aufhob, dass da etwas aus der Ordnung gekommen war und sich so ein Stück Freiheit von der Ordnung offenbarte... all das mögen Faktoren sein, die über das einfache Auslachen des alten Herrn S. hinausgingen. Das Signal des lachenden Menschen hat für jeden Beobachter eine aufmerksamkeitsregulierende Funktion. Es setzt die Neugier frei, die sich als Frage manifestiert, weshalb da gelacht wird. Ein zutiefst soziales Phänomen unterstreicht sich hier erneut: Hatte ich oben bemerkt, dass das Lachen ansteckend ist, wie ein aktueller Virus, so ist das Bild der Infektion eine gute Metapher für den sozialen Vorgang der kollektiven Einladung, ein gleiches zu tun. In unserem Beispiel wirkte der Virus bereits bei Michael und mir schon so intensiv, so dass wir uns gegenseitig in unserer Ansteckung stimulierten. Der Knabe, der ich war, wurde in eine zwischenmenschliche Handlung hineingezogen und wurde darin zu Mitmachen akti- 
viert. Oder war nicht doch ich der Antreiber gewesen? Heute weiß ich es nicht mehr oder habe es erfolgreich verdrängt und verschoben? In welchem Fall auch immer, es ist vom Abstand eines halben Jahrhunderts her nun eigentlich auch gleichgültig. Dass aber die theatrale Rolle des Verführers zum Lachen in der christlichen Lehre dem Satan zukommt, stimmt freilich bedenklich. Gleichwohl auch das: Wer will schon der Versucher sein!

Von unseren Beobachtern wurde die Infektion zum Lachen jedoch nicht angenommen. Hier unterscheidet sich dann auch das verbotene vom erlaubten und damit konsensualen Lachen. Das verbotene Lachen wurde von unseren Beobachtern in seiner Angemessenheit beurteilt und letztlich als nicht dem Wertekonsens des Lachhaften entsprechend abgelehnt. Die Ablehnung oder Zurückweisung eines unstatthaften Lachens kann von Empörung, Verurteilung bis zur sofortigen Unterbindung und Bestrafung reichen. Das Spektrum der Ablehnung ist breit gefasst und unterliegt zahlreichen Einflüssen. Es offenbart sich unversehens, dass das verbotene Lachen eine Waffe sein kann. In den zahlreichen und tiefgreifenden Auseinandersetzungen um satirische Kritik (Mohammed-Karikaturen) gilt das bis in die Gegenwart hinein.

Folgendes wäre ein weiteres Moment: Es ereignen sich Störungen ${ }^{4}$, die die Regeln des Rituals unterlaufen und sie teilweise in Frage stellen, vielleicht gar außer Kraft setzen oder noch schlimmer: sich als Gegenmacht etablieren. Da ist der fehlende Ernst der herangewachsenen Kinder, die sich in einer vorpubertären Leichtigkeit dem Respekt, den sie der Situation erweisen müssten, entziehen. Dass der Gottesdienst eine ernste Angelegenheit ist, steht natürlich außer Zweifel, bei Kindern jedoch nicht immer, dafür sind es eben Kinder, denen unversehens und ungerufen Dinge durch den Kopf und in die Glieder fahren, die sie „aus der Fassung“ bringen. Fassungslosigkeit, die Freude an Lächerlichem, der Spaß beim Tun verbotener Dinge, all das verweist auf Sündenfälle in Kinderzeiten. Der greise Herr S. hat sich davon auch nicht aus seiner Ruhe bringen lassen, was für seine gütige Welterfahrung und Weisheit spricht. Mit keinem Wort ging er oder andere - die Ausnahme der strafenden Hand habe ich ja erwähnt - auf unsere Verfehlung später ein und wir lebten weiter, als sei nichts gewesen. Das Fehlverhalten im Gottesdienst stellte gewiss einen Tabubruch dar, aber es erwies sich (zumindest für mich aus heutiger Sicht), dass gütige Toleranz wohl auch in solchen Fällen von Ketzerei eine bessere und überlegtere Reaktion darstellt, als gewalttätige Strafe.

4 Über die Theorie der Störung siehe: Carsten Gansel und Norman Ächtler, Hg., Das 'Prinzip Störung' in den Geistes- und Sozialwissenschaften (Berlin/Boston: Verlag de Gruyter, 2013). 
Zu den Störungen, die mit den Wahrnehmungen der Kinder zu erleben waren, gehört sicherlich der gebrechliche Gang und die besondere Mimik sowie mühselige Artikulation von Herrn S. Freilich wussten wir Kindern, dass man über alte, gebrechliche oder von Krankheit gezeichnete Menschen nicht lacht, aber es erging uns ja wie den drei Übeltätern in Heinrich Hoffmanns Struwwelpeter ${ }^{5}$, die in der Geschichte Von den schwarzen Buben über das „Mohrenkind“ unvermittelt und lauthals lachen mussten. Das Andere, das oft im Fremden zutage tritt, wird sehr schnell verlacht, sofern es nicht gleichzeitig so erschreckend anders ist, als dass es Angst erzeugt. Hier liegt ja auch die Nähe, die das Schreckliche im Komischen sichtbar werden lässt. Viele äußeren Eindrücke, die als neuartig, als bisher noch nie erblickt und erlebt worden sind, haben das Potential Angst zu erzeugen. Hier spielen sicherlich genetische Dispositionen in unserer Ausstattung eine nicht zu unterschätzende und bis heute wirksame Rolle. Nicht selten ruft diese Angst dann gewalttätige Handlungen der Abwehr hervor. Dass diese Angst aber auch im Lachen verdrängt wird, hat Freud 6 bereits beschrieben. Die im Struwwelpeter vorliegende rassistische Reaktion, die sich in Hohn, Spott, Häme und unbändigem Lachen entlädt, gibt zivilisatorisch tief zu denken, sie ist jedoch in der damaligen und heutigen Welt mit unabsehbaren Folgen in vielen Formen der Fremdenfeindlichkeit präsent. Die Angst, die vielleicht gar nicht weiter zu begründen ist, als dass das Neue, Fremde, Andere eben neu, fremd und anders ist, wirkt bis in die heutigen Tage hinein. Daher ist nur folgerichtig, dass die Angst bspw. vor Zuwanderern nicht durch Integrationskurse der Zugewanderten, sondern vielmehr durch Schulen der Angst bei den Autochthonen bekämpft werden sollte. Der Tragiker Heiner Müller fasst diese Angst auf seine Weise zusammen: „Die erste Gestalt der Hoffnung ist die Furcht, die erste Erscheinung des Neuen der Schrecken"7.

Eine weitere Seite der Störung besteht unzweifelhaft in der blasphemischen und gotteslästerlichen Handlung des Lachens. „Vor Gott wird nicht gelacht“, „der Glaube ist eine ernste Sache“, „man ist in der Kirche, im Hause Gottes ehrerbietig“... Man könnte die Reihe mit weiteren ungeschriebenen, aber nichts desto wirksamen Geboten füllen, an der Aussperrung des Lachens aus dem Gottesdienst ändert das nicht. Lachen ist der Häresie ver-

5 Heinrich Hoffmann, „Von den schwarzen Buben“, in Lustige Geschichten und drollige Bilder mit 15 schön kolorirten Tafeln für Kinder von 3-6 Jahren (Frankfurt am Main: Literarische Anstalt, 1845); volkstümlich später genannt: Der Struwwelpeter.

6 Vgl. Sigmund Freud, Der Witz und seine Beziehung zum Unbewußten (Leipzig/Weimar: Gustav Kiepenheuer Verlag, 1989).

7 Heiner Müller, „Der Schrecken die erste Erscheinung des Neuen. Zu einer Diskussion über Postmodernismus in New York“, in Heiner Müller, Werke 8. Schriften (Frankfurt am Main: Suhrkamp Verlag, 2005), 208-212. 
gleichbar, es ist Teufelswerk und daher in jedem Falle aus dem Hause zu bannen. Die etwas altertümlichen Formulierungen verraten die Überzeitlichkeit dieses ethisch-moralischen Fehlverhaltens, das unter historischem Vorzeichen in kirchlich verfassten politischen Ordnungen streng gemaßregelt wurde. Dass zumindest in der christlich-europäischen Tradition das verbotene Lachen dem satanischen Höllengeistern zugeordnet wurde, hieße ein weiteres Kapitel aufschlagen, das ebenso unterbleiben muss, wie das Lachen unter den kommunikativen Aspekten der Kunstausübung. Hier tun sich komplexe Felder auf, die hier nicht einmal angedeutet werden sollen.

\title{
References
}

Bachorski, Hans-Jürgen. "Poggios Facetien und das Problem der Performativität des toten Witzes." Paragrana, no. 10 (2001): 318-335.

Freud, Sigmund. Der Witz und seine Beziehung zum Unbewußten. Leipzig/Weimar: Gustav Kiepenheuer Verlag, 1989.

Gansel, Carsten, and Norman Ächtler, ed. Das "Prinzip Störung" in den Geistesund Sozialwissenschaften. Reihe: Studien und Texte zur Sozialgeschichte der Literatur 133. Berlin/Boston: Verlag de Gruyter, 2013.

Hoffmann, Heinrich. "Von den schwarzen Buben." In Hoffmann, Heinrich. Lustige Geschichten und drollige Bilder mit 15 schön kolorierten Tafeln für Kinder von 3-6 Jahren. Frankfurt am Main: Literarische Anstalt, 1845.

Müller, Heiner. "Der Schrecken die erste Erscheinung des Neuen. Zu einer Diskussion über Postmodernismus in New York." In Müller, Heiner. Werke 8. Schriften, 208-212. Frankfurt am Main: Suhrkamp Verlag, 2005. Semjonowitsch Makarenko, Anton. Der Weg ins Leben. Ein pädagogisches Poem. Berlin: Aufbau Verlag, 1950.

Sonntägliche Kinderhandlung. https://anthrowiki.at/Der_freie_christliche_Impuls\#Sonntagshandlung_f.C3.BCr_die_Kinder.5B31.5D.

\section{Über das verbotene Lachen}

\begin{abstract}
Der Essay geht der Frage nach, was passiert, wenn das Lachen in verbotenen Kontexten sich nicht unterdrücken lässt. Ein Kindheitserleben ist der Anlass über die kommunikativen Verhältnisse des verbotenen Lachens nachzudenken. Es wird dabei auf das Lachen als ein besonderes kommunikatives Zeichensystem und körperliches Ereignis hingewiesen. Schlüsselwörter: Theater, Phylogenese, Tabu, Strafen, Beobachtungen (Luhmann), Karneval, Häme.
\end{abstract}




\section{O zakazanym śmiechu}

Abstrakt: Tematem eseju jest specyficzna sytuacja komunikacyjna powstała w wyniku wy-
buchu niedającego się stłumić śmiechu w zakazanych kontekstach. Doświadczenie z dzieciń-
stwa stało się okazją do refleksji nad uwikłaniem komunikacyjnym zakazanego śmiechu. Jed-
nocześnie podjęta została próba charakterystyki śmiechu jako specyficznego systemu zna-
ków służących komunikacji oraz jako aktu czysto cielesnego.
Słowa kluczowe: teatr, filogeneza, tabu, kara, obserwacje (Luhmann), karnawał, zjadliwość. 